\title{
A tale of two risks: smoking, diabetes and the subgingival microbiome
}

\author{
Sukirth M Ganesan ${ }^{1}$, Vinayak Joshi², Megan Fellows ${ }^{1}$, Shareef M Dabdoub ${ }^{1}$, \\ Haikady N Nagaraja ${ }^{3}$, Benjamin O’Donnell ${ }^{4}$, Neeta Rohit Deshpande ${ }^{5}$ \\ and Purnima S Kumar ${ }^{1}$ \\ ${ }^{1}$ Division of Periodontology, College of Dentistry, The Ohio State University, Coloumbus, OH, USA; ${ }^{2}$ Maratha \\ Mandal's NathajiRao G. Halgekar Institute of Dental Sciences and Research Center, Rajiv Gandhi University, \\ Belgaum, India; ${ }^{3}$ College of Public Health, The Ohio State University, Coloumbus, OH, USA; ${ }^{4}$ Division of \\ Endocrinology, Diabetes and Metabolism, The Wexner Medical Center, The Ohio State University, \\ Coloumbus, OH, USA and ${ }^{5}$ Department of General Medicine, Maratha Mandal's Nathajirao G. Halgekar \\ Institute of Dental Sciences \& Research Centre, Rajiv Gandhi University, Belgaum, India
}

\begin{abstract}
Although smoking and diabetes have been established as the only two risk factors for periodontitis, their individual and synergistic impacts on the periodontal microbiome are not well studied. The present investigation analyzed 2.7 million 16S sequences from 175 non-smoking normoglycemic individuals (controls), smokers, diabetics and diabetic smokers with periodontitis as well as periodontally healthy controls, smokers and diabetics to assess subgingival bacterial biodiversity and co-occurrence patterns. The microbial signatures of periodontally healthy smokers, but not diabetics, were highly aligned with the disease-associated microbiomes of their respective cohorts. Diabetics were dominated by species belonging to Fusobacterium, Parvimonas, Peptostreptococcus, Gemella, Streptococcus, Leptotrichia, Filifactor, Veillonella, TM7 and Terrahemophilus. These microbiomes exhibited significant clustering based on HbA1c levels (pre-diabetic $(<6.5 \%)$, diabetic $(6.5-9.9 \%)$, diabetics $>10 \%)$. Smokers with periodontitis evidenced a robust core microbiome (species identified in at least $80 \%$ of individuals) dominated by anaerobes, with inter-individual differences attributable largely to the 'rare biosphere'. Diabetics and diabetic smokers, on the other hand, were microbially heterogeneous and enriched for facultative species. In smokers, microbial co-occurrence networks were sparse and predominantly congeneric, while robust inter-generic networks were observed in diabetics and diabetic smokers. Smoking and hyperglycemia impact the subgingival microbiome in distinct ways, and when these perturbations intersect, their synergistic effect is greater than what would be expected from the sum of each effect separately. Thus, this study underscores the importance of early intervention strategies in maintaining health-compatible microbiomes in high-risk individuals, as well as the need to personalize these interventions based on the environmental perturbation.

The ISME Journal (2017) 11, 2075-2089; doi:10.1038/ismej.2017.73; published online 23 May 2017
\end{abstract}

\section{Introduction}

Periodontitis, a polymicrobial disease that causes destruction of the structures that anchor the tooth to the jawbone, is the sixth most prevalent disease in the world, affecting over 750 million people (Eke et al., 2012; Kassebaum et al., 2014). Smoking and diabetes are the only known risk factors for this disease, increasing the extent and severity of periodontitis by 3-10-fold and hastening periodontal destruction exponentially (1996; Bergstrom et al.,

Correspondence: PS Kumar, Division of Periodontology, College of Dentistry, The Ohio State University, 4111 Postle Hall, 305, W 12th Avenue, Columbus, OH 43210, USA.

E-mail: kumar.83@osu.edu

Received 20 October 2016; revised 6 March 2017; accepted 22 March 2017; published online 23 May 2017
2000; Tomar and Asma, 2000). Periodontitis has been called the sixth complication of diabetes (Loe, 1993), with one-third of diabetics suffering from severe periodontitis (CDC, 2014). Nearly $42 \%$ of periodontitis cases can be attributed to smoking (Tomar and Asma, 2000), and critically, 24\% of diabetics smoke (Ford et al., 2004), thus creating three high-risk groups for periodontal diseases: smokers, diabetics and diabetic smokers. With nearly 30 million Americans suffering from adult-onset diabetes (CDC, 2014) and over 1 billion smokers worldwide (Ng et al., 2014), preventing periodontitis in these high-risk populations will be a highly cost effective healthcare strategy.

Although it is established that dysbiosis of the indigenous periodontal microbiome is the primary etiological trigger for periodontitis 
(Griffen et al., 2012), the events that lead to this dysbiosis are not well understood. A central characteristic of any ecosystem is its response to environmental perturbations, which plays a critical role in altering both community structure and membership. Smoking and diabetes are two environmental presses that affect the subgingival environment in unique ways. While smoking leads to peripheral vasoconstriction (Clarke et al., 1981), decreased vascular permeability and hyperemia (Mavropoulos et al., 2003), decreased flow of gingival crevicular fluid (Gomes et al., 2009) and lower oxygen tension (Hanioka et al., 2000), hyperglycemia affects this environment by increasing glucose levels (Yamaguchi et al., 2005), vascular permeability, and levels of matrix metallo-proteinases, cytokines and adhesion molecules (reviewed by Taylor et al., 2013). Thus, the subgingival milieu in smokers is anaerobic, acidic, reducing, nutritionally deprived and immune-impaired. In diabetics, this environment is glucose-rich, pro-oxidant, protein-rich and anaerobic.

The effects of smoking on the oral microbiome have been extensively studied in recent years and several investigations have established that the oral microbiome shifts in response to this environmental press (Fullmer et al., 2009; Delima et al., 2010; Shchipkova et al., 2010a; Kumar, 2012; Wu et al., 2016). Smoking creates pathogen-rich, commensalpoor microbial ecosystems even in clinically healthy individuals that closely resemble diseaseassociated communities (Kumar et al., 2011a; Mason et al., 2015). Although it is known that uncontrolled hyperglycemia similarly increases the risk for periodontitis, very few studies have examined the effects of diabetes on the subgingival microbiome (Zambon et al., 1988; Ciantar et al., 2005; Larsen et al., 2010; Ohlrich et al., 2010; Casarin et al., 2013; Zhou et al., 2013). Furthermore, there are no studies that examine the effects of intersecting environmental presses on periodontal microbiota.

Hence, the goal of the present investigation was to examine the effects of each environmental press on the structure and membership of the subgingival microbial communities using an open-ended deep sequencing approach. To the best of our knowledge, we present the first available evidence on the combined effects of these two environmental presses on the subgingival microbiome.

\section{Materials and methods}

\section{Ethics statement}

Approval for this study was obtained from the Office of Responsible Research Practices at The Ohio State University and the Ethics Committee of Rajiv Gandhi University and carried out according to the approved guidelines. All participants gave written informed consent prior to enrollment.

\section{Subject selection}

Subjects with generalized moderate to severe chronic periodontitis (attachment loss $\geqslant 5 \mathrm{~mm}$, probing pocket depths $\geqslant 5 \mathrm{~mm}$, mean gingival index (Loe and Silness, 1963) $>1$ in $30 \%$ of more of sites (Armitage, 1999)) were recruited from two centers in India and the United States, and informed consent obtained. A total of 25 subjects were recruited into each of the following groups: normoglycemic nonsmokers, hyperglycemic nonsmokers, normoglycemic smokers and hyperglycemic smokers. Additionally, 75 periodontally healthy individuals were recruited and distributed equally among the three groups: normoglycemic nonsmoker, hyperglycemic nonsmoker and normoglycemic smoker. Periodontal health was defined as attachment loss $\leqslant 1 \mathrm{~mm}$, probing pocket depths $\leqslant 3 \mathrm{~mm}$, mean gingival index $<1$. Smokers were defined as $\geqslant 10$ packyear histories and currently smoking more than 3 times a week, nonsmokers were defined as those who smoked $<100$ cigarettes in their lifetime and were not currently smoking (CDC guidelines). Type 2 diabetes was defined as untreated adult-onset hyperglycemia with a glycated hemoglobin (HbA1c) level of $\geqslant 6.5$ and normoglycemia as HbA1c of $\leqslant 6$ (American Diabetes Association, 2015). Exclusion criteria included conditions that required the use of prophylactic antibiotics, current or planned pregnancy, HIV infection, long-term ( $>3$ months) use of medications known to cause gingival changes, (for example, immunosuppressants, phenytoin, calcium channel blockers, aspirin, NSAIDS, bisphosphonates or steroids), antibiotic therapy or oral prophylactic procedures within the last 3 months and less than 20 teeth in the dentition. The groups were frequency matched for age, gender, BMI and extent and severity of periodontal destruction.

\section{Sample collection}

Samples were collected from both deep and shallow sites of subjects with periodontitis. Prior to sample collection, selected sites were isolated and supragingival plaque was removed. Subgingival plaque was collected and pooled from four non-adjacent proximal sites with probe depths of $\geqslant 6 \mathrm{~mm}$, BOP, and GI $>1$ (disease or deep-site samples) by inserting 15 sterile endodontic paper-points (CaulkDentsply, Milford, DE, USA). Samples were similarly acquired from four sites with probe depths of $\leqslant 3$, no BOP and GI $\leqslant 1$ and separately pooled (healthy or shallow-site samples). A total of 15 randomly selected interproximal sites were sampled from periodontally healthy subjects. Samples were placed in 1.5-ml microcentrifuge tubes and frozen at $-20^{\circ} \mathrm{C}$ until further analysis.

Sequencing and analysis pipeline

DNA was isolated as previously described (Kumar et al., 2011b) and multiplexed bacterial tag-encoded 
FLX 16S pyrosequencing was performed using the Titanium platform (Roche Applied Science, Indianapolis, IN, USA). Two regions of the 16S rRNA genes were sequenced: V1-V3 (spanning E.coli 16S gene regions 8-27 and 519-536) and V7-V9 (spanning E.coli 16S gene regions 1099-1114 and 1528-1541). The primers used for sequencing have been previously described (Kumar et al., 2011b). Each primer is capable of detecting a range of genera that the other fails to recover. Together they allow the recovery of a wider range of the microbiome than is possible with a single primer alone. However, some genera are picked up by both primers. Thus, to prevent over counting, the number of sequences assigned to an OTU by both primers was reduced by half. Primer averaging was carried out as previously described (Kumar et al., 2011b) using the implementation in the PhyloTOAST software suite (Dabdoub et al., 2016). Sequences with an average quality score of 30 over a sliding window of $50 \mathrm{bp}$ and length $>200 \mathrm{bp}$ were assigned a taxonomic identity by alignment to the HOMD database (Chen et al., 2010) using the Blastn algorithm. Analyses were conducted using the QIIME (Caporaso et al., 2010) and PhyloToAST (Dabdoub et al., 2016) pipelines.

\section{Statistical analysis}

Principal coordinate analysis (PCoA) of UniFrac distances was used to interrogate the robustness of group-wise clustering and Adonis test was used to estimate statistical significance of group-wise betadiversity (McArdle and Anderson, 2001). Linear discriminant analysis (LDA) was performed using the MASS package for R. The input for LDA was a matrix of variance-stabilized (arc-sin square root transformed) relative abundances of species-level OTUs (Shchipkova et al., 2010b). MASS:lda provided singular value decomposition (svd) values, which were used to calculate the percent variance explained in each dimension. MANOVA/Wilks lambda was used to test for significance of LDA clustering. Alpha diversity of s-OTUs was estimated using Abundance Coverage Estimator (ACE) Index and the Wilcoxon and Kruskal-Wallis tests were used for significance testing of two and multi-group comparisons respectively. Differential abundance analysis of OTUs was carried out using DESeq2 (Love et al., 2014) and $P$-values adjusted for multiple testing (FDR $<0.1$, FDR-adjusted Wald Test). Results were visualized using PhyloToAST (Dabdoub et al., 2016; PCoA plots, diversity curves), and the Interactive Tree Of Life webserver (phylogenetic tree) (Letunic and Bork, 2011). Species-level co-occurrence networks for each group were created using JMP (SAS Institute Inc., Cary, NC, USA) to calculate pairwise correlations; significant co-occurrences (defined as Spearman's rho $>0.75$ and $P<0.05$ ( $t$ test of rho)) were imported into Networkx (Hagberg et al., 2008) to create the graph structures, and Gephi (Bastian et al., 2009) to visualize and label the graphs. A core microbiome was computed for each group as the suite of species identified in $\geqslant 80 \%$ of individuals in that group. Robustness of clustering was examined using an algorithm incorporating betweenness centrality, differential abundances, and frequency of occurrence in a group as described before (Paropkari et al., 2016). Betweenness centrality was calculated using Python package 'Networkx' and s-OTUs were ranked based on this metric. For each group, significantly different $(P<0.05$, TukeyHSD) species were identified using JMP (SAS Institute Inc.) and species that were present in at least $75 \%$ of the subjects were identified using QIIME's core_microbiome.py script. Species that demonstrated a high betweenness centrality (top $20 \%$ in each network), and were either part of the group's core microbiome or showed significant differences between the groups were identified as network anchors. A relative risk model was used to investigate whether the effects of smoking and diabetes were additive or multiplicative (Hammond et al., 1979). Contingency tables were created using smoking and diabetes as conditions and alpha diversity, relative abundances of gram-positive and gram-negative organisms and the number of species in the core microbiome as response variables. Relative risk was calculated for each response variable in diabetics and smokers and summed to compute the expected relative risk when both factors intersect. This was compared to the observed relative risk in diabetic smokers.

\section{Results}

Table 1 shows the demographic, clinical and biometric characteristics of the groups. A total of 2.7 million high quality, classifiable sequences from 275 samples were used for analysis. The sequences have been deposited in the Sequence Read Archive of the NCBI (Accession number: SRP090878). These sequences represented 564 species-level operational taxonomic units (s-OTUs) with an average of $168 \pm 54$ s-OTUs in each sample. The samples did not demonstrate any clustering based on geographic location (India versus United States, data not shown).

Periodontitis is associated with a global dysbiosis of the subgingival microbial ecosystem

Chronic periodontitis is a site-specific disease, that is, at any point in time certain teeth demonstrate destruction of tooth-supporting structures and inflammation (deep sites) and others are diseasefree (shallow sites; Goodson et al., 1982). Principal coordinate analysis (PCoA) of unweighted UniFrac distances did not show significant clustering of sites $(P=0.76)$ based on this clinical phenotype (Figures 1a-d). The ACE index was not significantly different between the clinical sites $(P=0.192$, 


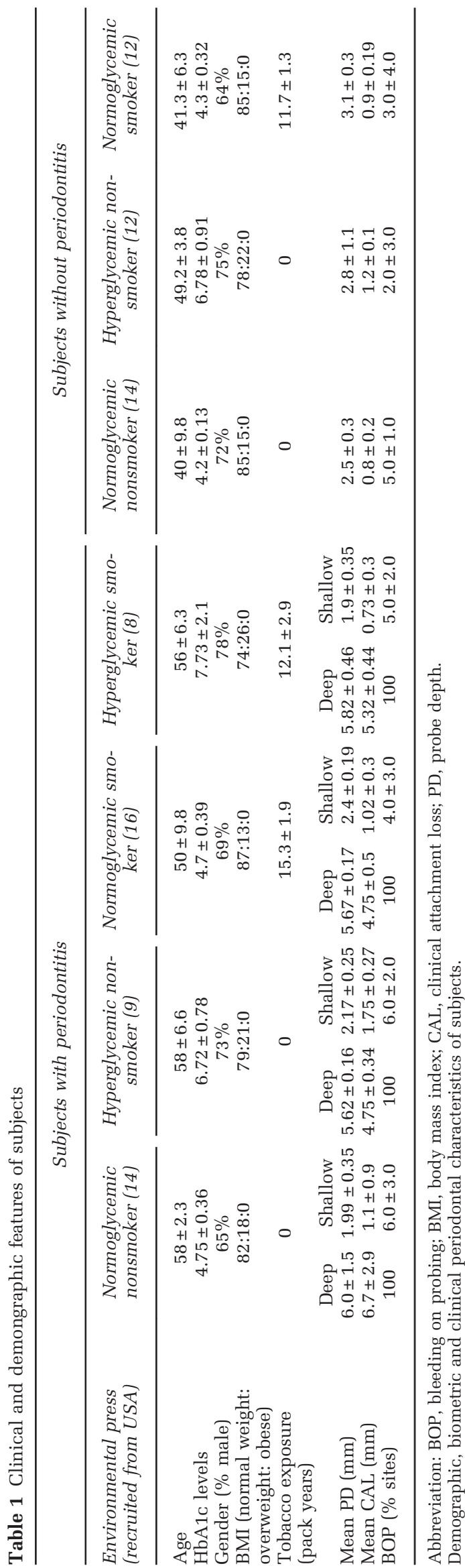

Figures 1e-h). Thus, in a subject with disease, differences in community structure or membership were not apparent based on severity of periodontal destruction.

Distinct subgingival microbial assemblages are associated with uncontrolled chronic hyperglycemia $75 \%$ of the microbiome of type 2 diabetics with periodontitis was composed of gram-positive facultatives and gram-negative anaerobes (Figure 2d). The community was dominated by species belonging to the genera Fusobacterium, Parvimonas, Peptostreptococcus, Gemella, Streptococcus, Leptotrichia, Filifactor, Veillonella, TM7 and Terrahemophilus.

When this community was compared to normoglycemic individuals with periodontitis, both PCoA ( $P=0.001$, Adonis test of UniFrac distances) and LDA $(P=0.001$, MANOVA Wilks test $)$ revealed a significant clustering of microbiomes based on glycemic status (Figure 2a). Moreover, within the diabetic group, significant clustering was observed based on HbA1c levels (pre-diabetic $(<6.5 \%)$, diabetic (6.5-9.9\%), diabetics $>10 \%$ (American Diabetes Association, 2015), Figure 2b). Diabetics exhibited significantly lower species richness $(P=0.0008$, Wilcoxon test Figure 2c), and lower levels of anaerobes and higher levels of facultatives (both gram-positive and gram-negative) when compared to normoglycemic individuals with periodontitis $(P<0.05$, Wilcoxon test Figure 2d), a finding that corroborates earlier research using a similar methodology (Casarin et al., 2013; Zhou et al., 2013).

A total of 81 species comprised the core microbiome of normoglycemic controls, 46 of these were not identified in hyperglycemic cores (Figure 3a). Also, only 42 species formed the core microbiome of periodontitis in diabetics and seven of these were not detected in normoglycemics with periodontitis.

Higher levels of species belonging to Lactobacillus, Corynebacterium, Pseudomonas and lower levels belonging to Treponema, Porphyromonas, Prevotella, and Parvimonas were observed in diabetics $(P<0.05$, FDR-adjusted Wald test, Figure $4 \mathrm{a})$.

Network analysis revealed significant cooccurrence patterns between the core species (Figure 5). In the normorglycemic state, cooccurrence was mainly congeneric and sparse, with species belonging to Streptococcus, Neisseria, Selenomonas and Treponema driving the clusters. On the other hand, diabetics with periodontitis displayed two robust microbial hubs with several intergeneric networks, one that consisted of uncultivated phylotypes belonging to TM7, Treponema, Microbacterium and Sphingomonas and the other comprising Fretibacterium and Peptostreptococcaceae [XI] [G4] and [XII] [G5], and Filifactor alocis.

To examine the ecological shifts from health to disease in hyperglycemics, the microbiomes of diabetics with and without periodontitis were analyzed and compared to periodontally healthy 

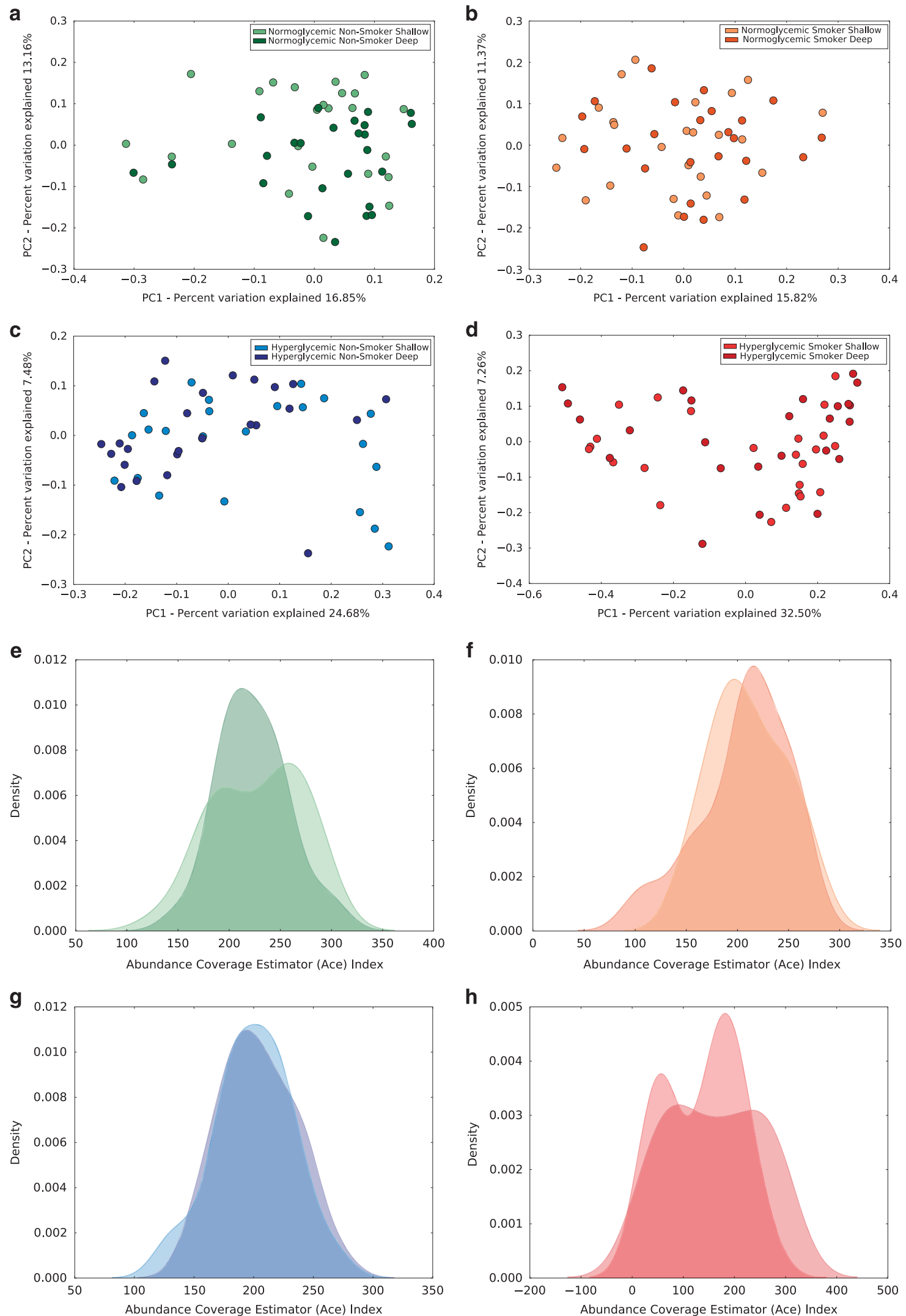

Figure 1 Similarity in microbial community configuration between deep and shallow sites. (a-d) show the principal coordinate analysis (PCoA) of UniFrac distances between deep and shallow sites in normoglycemic nonsmokers, normoglycemic smokers, hyperglycemic nonsmokers and hyperglycemic smokers. (e-h) show the kernel density plots of alpha diversity (ACE) between the same sites in the same individuals. The peak indicates the median values for each group, and the $x$ axis shows the data range. Neither the PCoA nor the ACE values differed significantly between deep and shallow sites. 

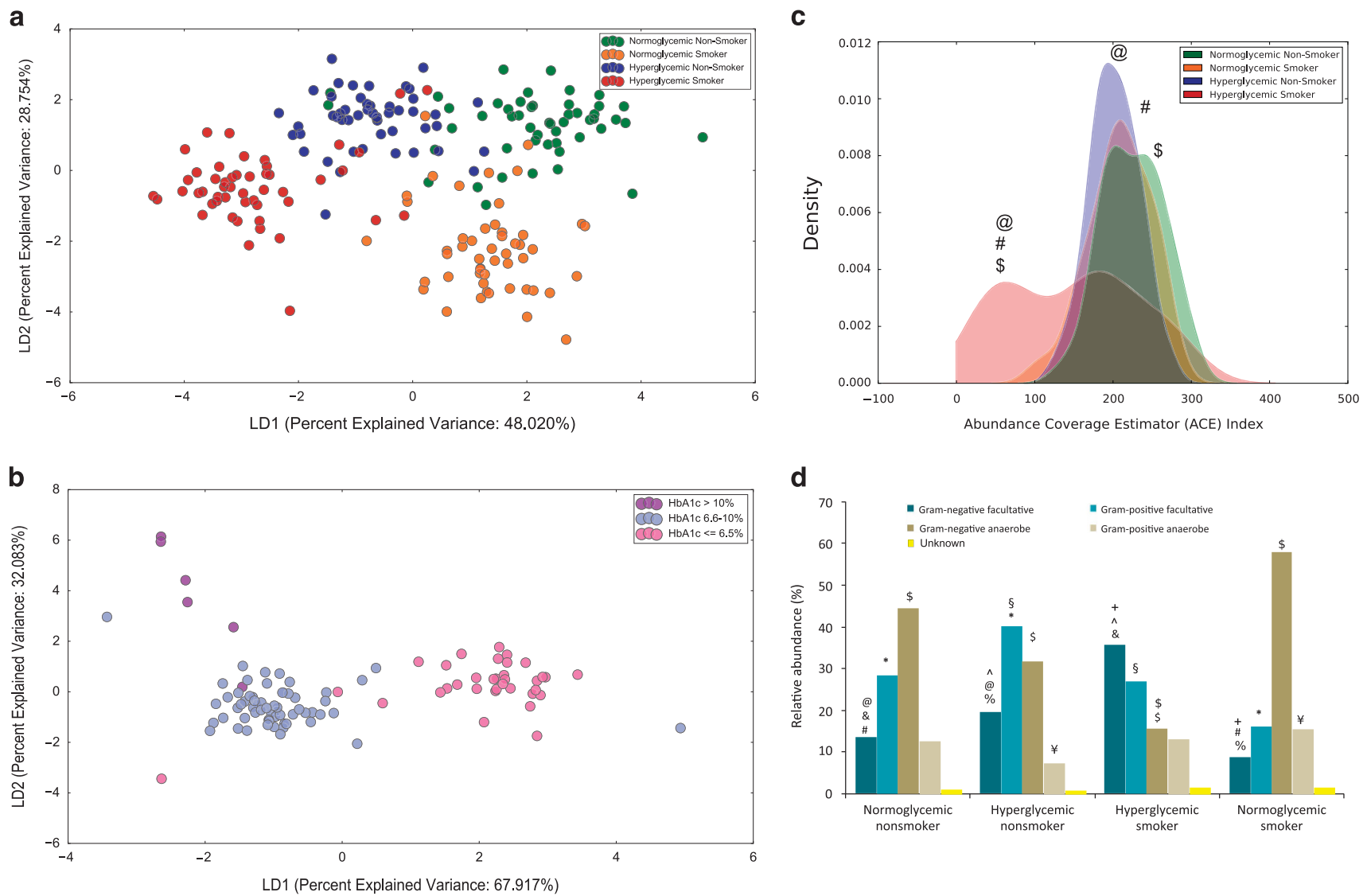

Figure 2 Differences in alpha and beta diversity metrics between the four groups. Linear discriminant analysis (LDA) of relative abundances of species-level operational taxonomic units (s-OTUs) in normoglycemic nonsmokers, normoglycemic smokers, hyperglycemic nonsmokers and hyperglycemic smokers with periodontitis is shown in (a) $(P=0.008$, MANOVA Wilks test). LDA of diabetics clustered by glycemic control is shown in $(\mathbf{b})(P=0.001)$. Density curves of alpha diversity $(\mathrm{ACE})$ are shown in $(\mathbf{c})(\mathrm{The}$ peak indicates the median values for each group, and the $x$ axis shows the data range) and distribution of s-OTUs by gram staining characteristics and oxygen requirements in (d). Groups that share the same symbol are significantly different from each other $(P<0.05$, Kruskal-Wallis test).

normoglycemics. PCoA and LDA revealed significant clustering of groups based on both glycemic status and periodontal health $(P=0.01$, Figure 6a). Periodontally healthy diabetics exhibited significantly lower species richness than both diabetics with periodontitis and periodontally healthy controls $(P=0.0019$, Figure 6b). Lower levels of gram-positive facultatives and higher levels of gram-positive and gram-negative anaerobes were seen in periodontally healthy hyperglycemics when compared to periodontally healthy normoglycemics (Figure 6c). When the data were analyzed at the level of the core species, further differences between periodontal health and periodontitis in diabetics could be observed (Figure $3 \mathrm{~b}$ ). Ten percent ( 7 out of 71 species) of the overall core microbiome (Campylobacter gracilis, Corynebacterium matruchotii, Fusobacterium nucleatum, Gemella sanguinis, Granulicatella adiacens, Streptococcus oralis and Streptococcus sanguinis) was shared by all the three groups. Forty-three percent (13 out of 30) of the species identified in periodontally healthy normoglycemics were not detected in periodontally healthy diabetics. In addition, 30 species were identified only in the core microbiome of periodontally healthy diabetics; however, none of these were detectable in diabetics with periodontitis (Figure 3b). Thus, it appears that a disease-associated community framework (with decreases in relative abundances of health-compatible species, and increases in levels of species belonging to the genera Porphyromonas, Prevotella, Campylobacter and Fusobacterium), is established in states of periodontal health in diabetics; and during progression to disease, community membership undergoes further shifts.

Distinct patterns of microbial assembly observed in smokers and hyperglycemics

PCoA and LDA revealed a significantly distinct clustering of periodontitis-associated microbiomes based on glycemic status and tobacco exposure $(P=0.001$, Figure 2a). Diabetics and smokers exhibited similar species richness $(P=0.3130$, Figure 2c). However, diabetics demonstrated lower levels of anaerobes and microaerophiles (belonging to the genera Parvimonas, Prevotella, Tannerella, Lachnoanaerobaculum, Lactobacillus, Fretibacterium, 
a

Normoglycemic Non-Smoker $\quad$ Normoglycemic Smoker

Hyperglycemic Non-Smoker

Hyperglycemic Smoker
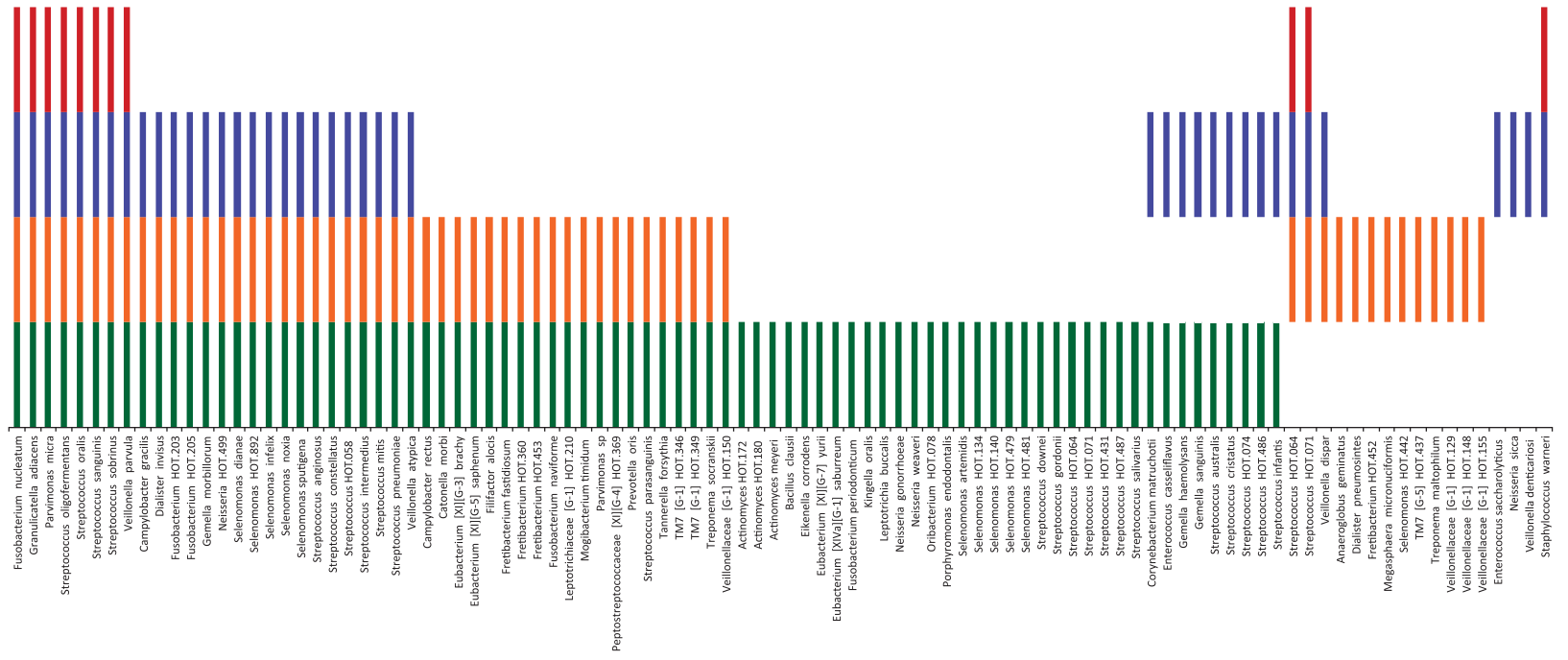

b

Periodontally healthy normoglycemics Periodontally healthy hyperglycemics

Hyperglycemics with periodontitis

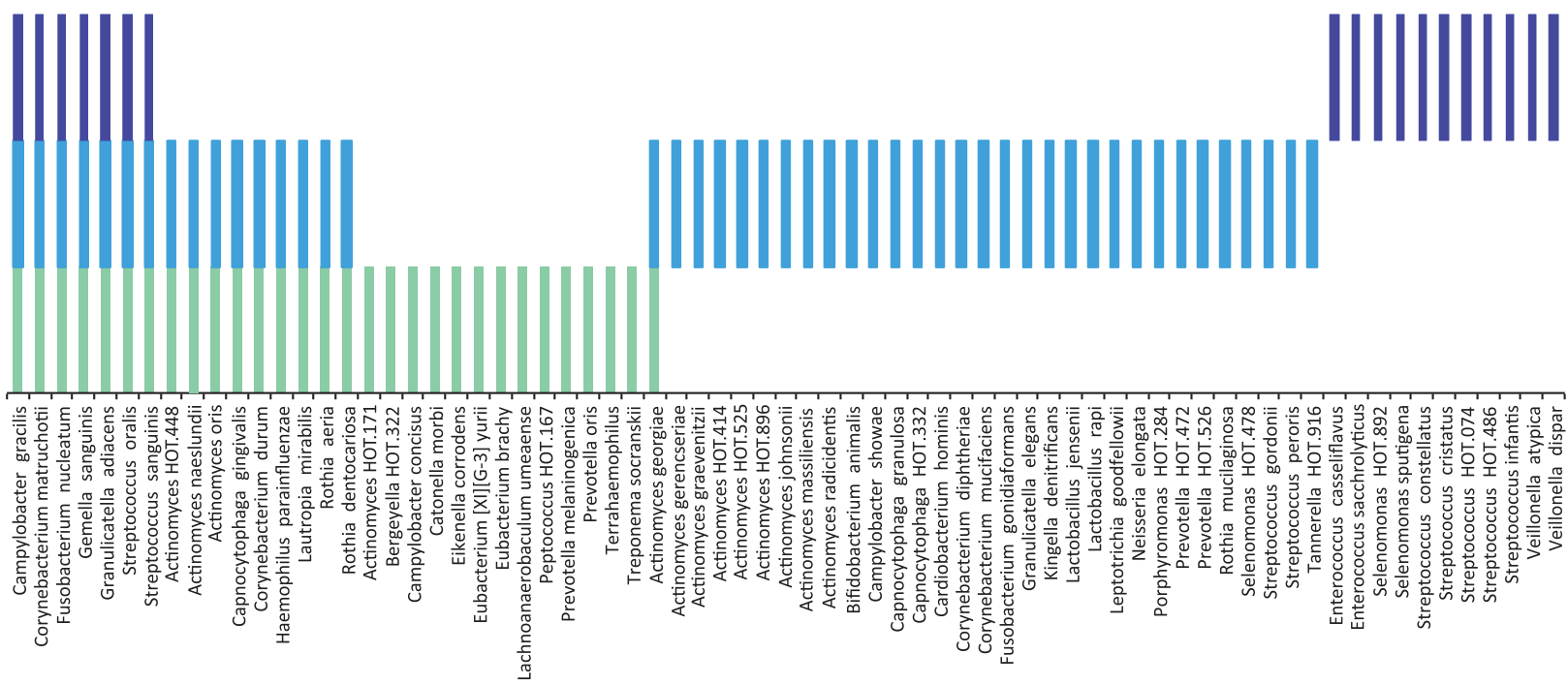

C

Periodontally healthy Smoker

Smokers with periodontitis

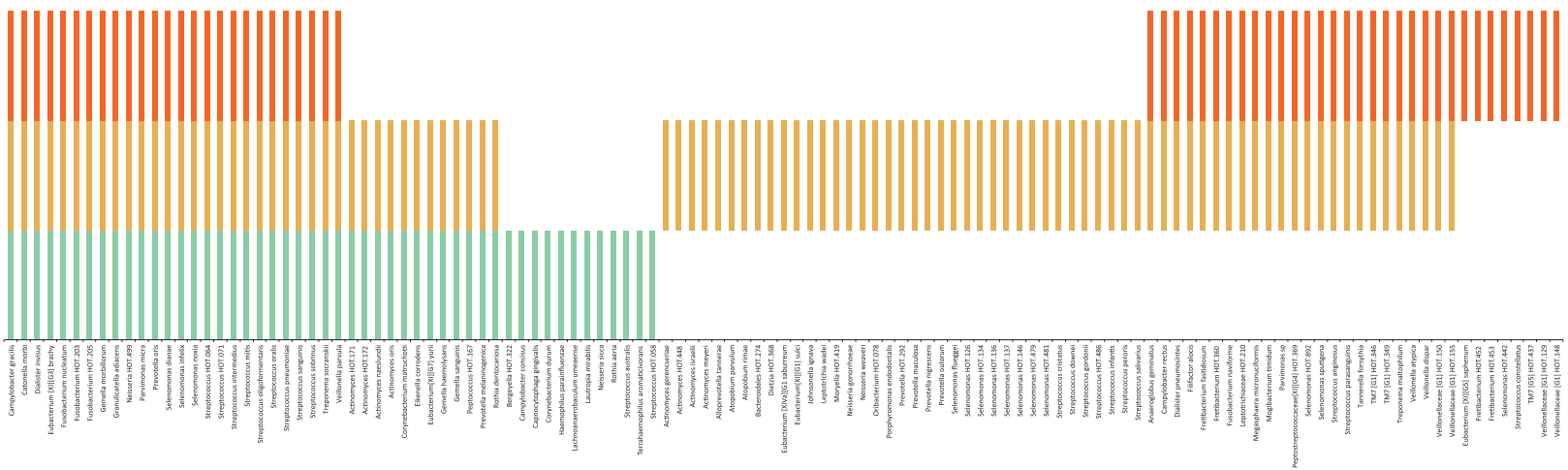

Figure 3 Core microbiome of normoglycemic nonsmokers, normoglycemic smokers, hyperglycemic nonsmokers and hyperglycemic smokers. (a) s-OTUs that were identified in the core microbiome of the four groups with periodontitis. (b) core microbiomes of periodontally healthy normoglycemics, periodontally healthy hyperglycemics and hyperglycemics with periodontitis. (c) core microbiomes of periodontally healthy nonsmokers, periodontally healthy smokers and smokers with periodontitis. 
a
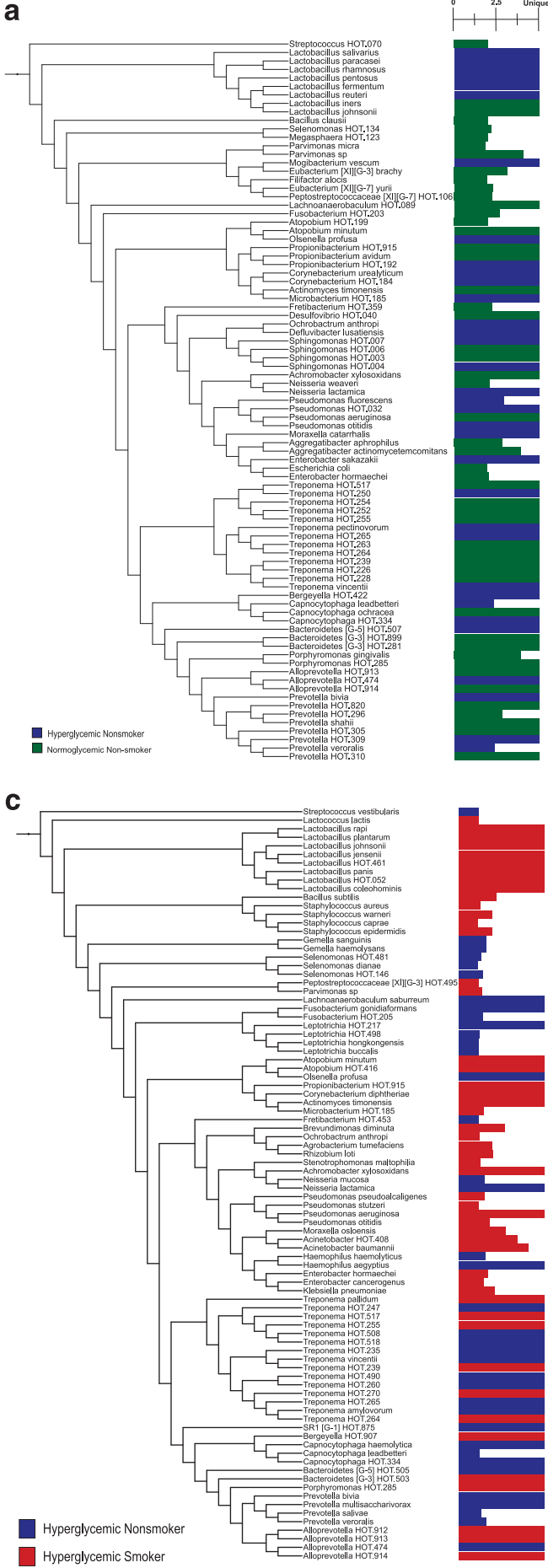

b

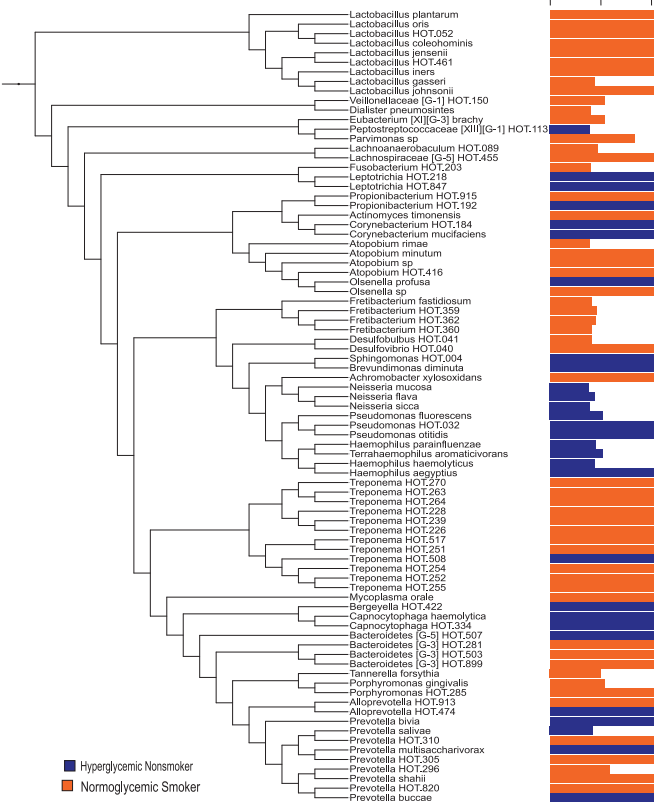

d

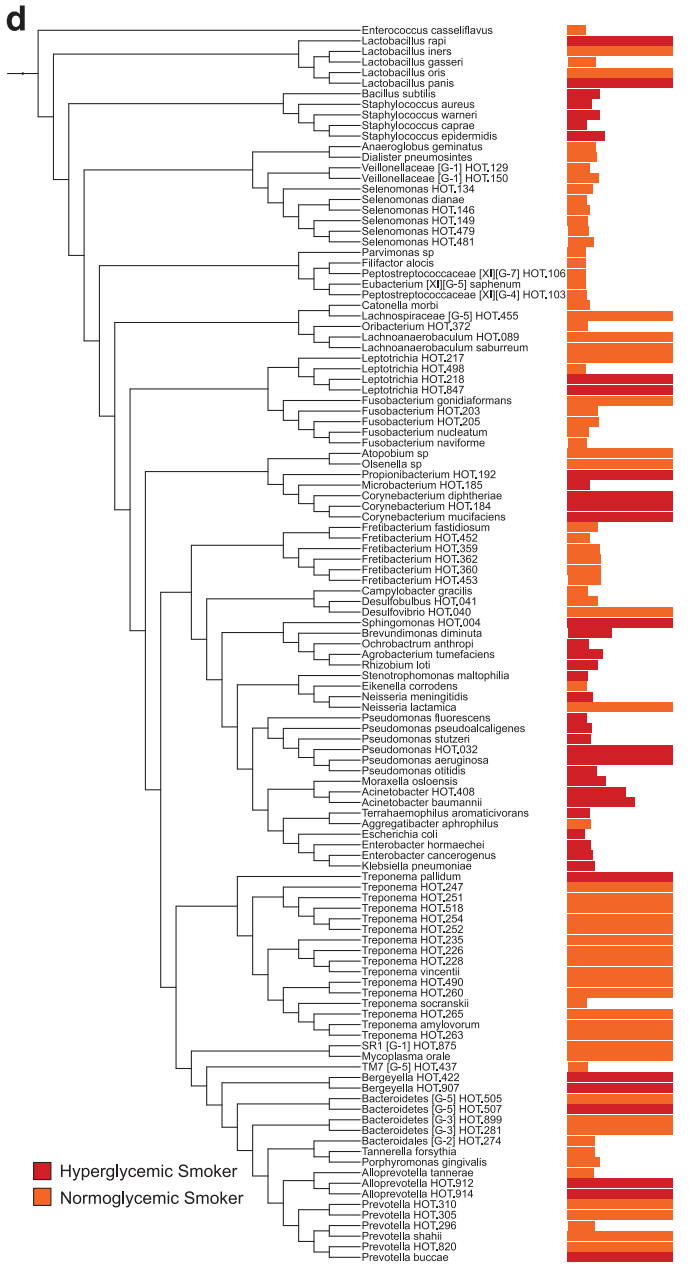

Figure 4 Species that were uniquely identified in normoglycemic nonsmokers, normoglycemic smokers, hyperglycemic nonsmokers and hyperglycemic smokers with periodontitis, as well as those that were significantly different between groups $(P<0.05$, FDR-adjusted Wald Test, DE-Seq2). Species are arranged by phylogeny and the fold differences (log2 scale) are shown. 


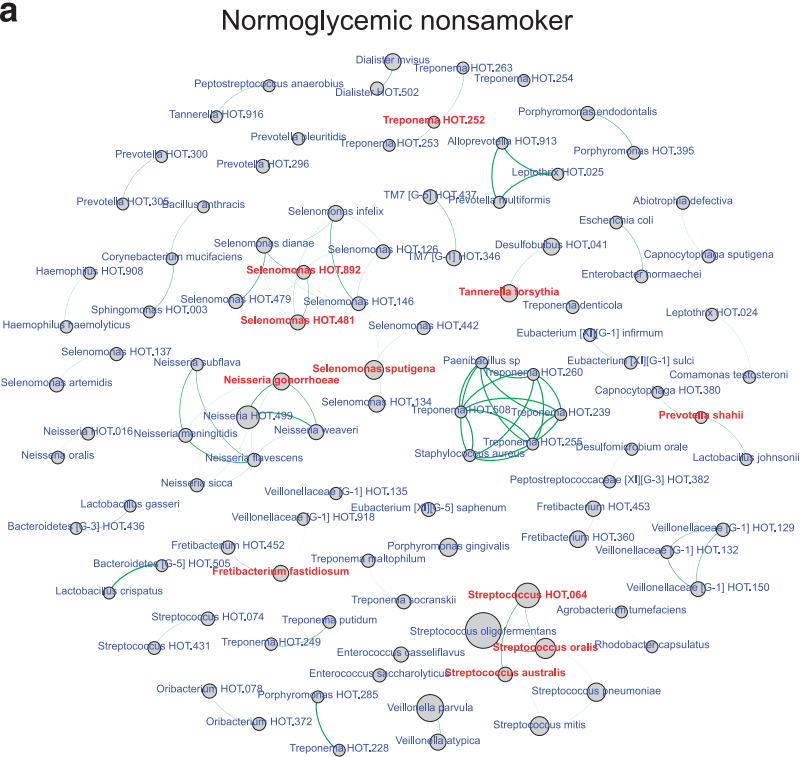

C

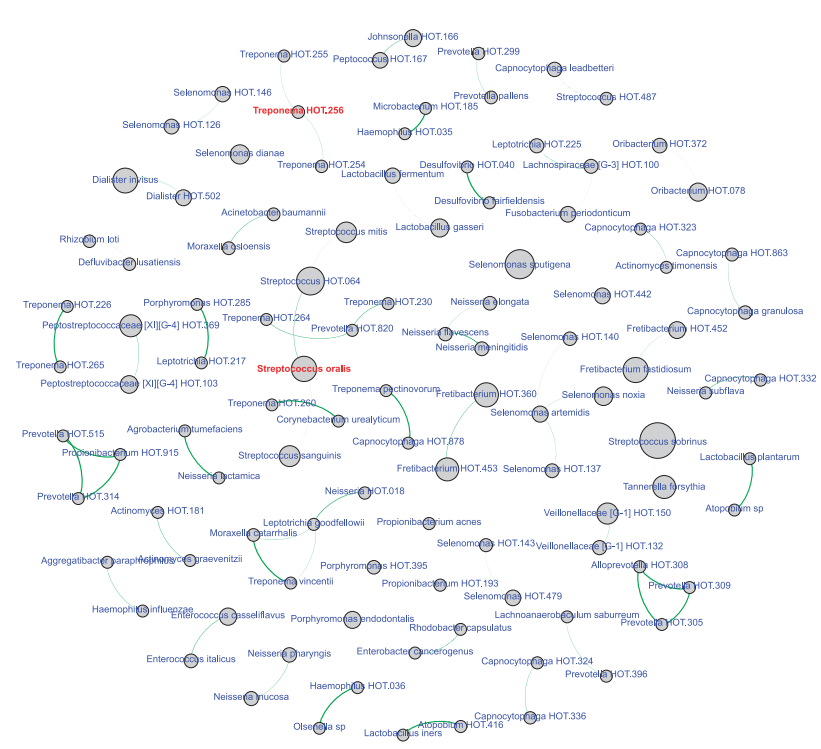

b

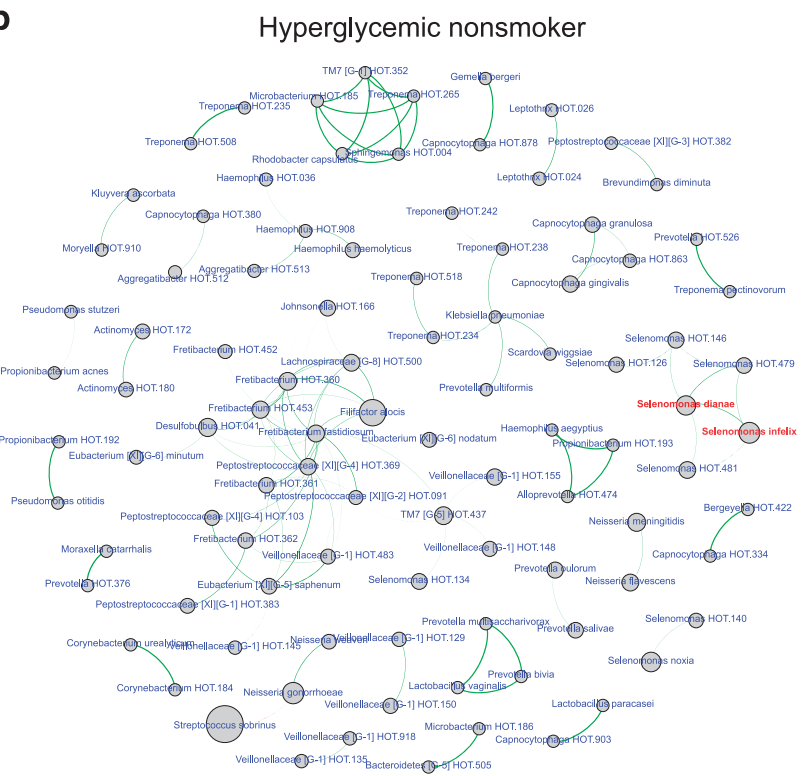

d

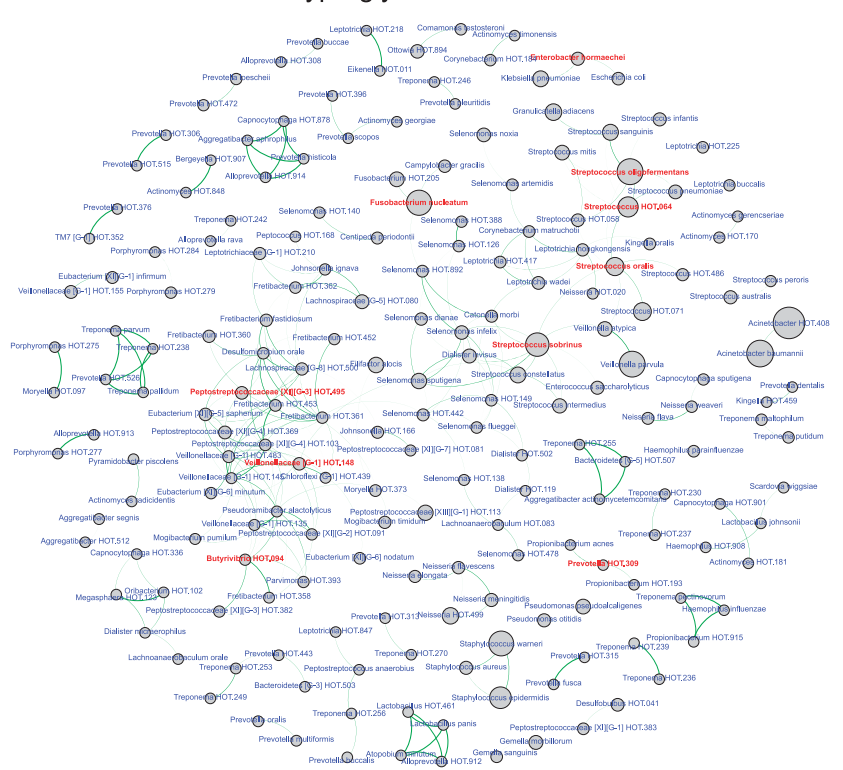

Figure 5 Co-occurrence networks in each group. Normoglycemic nonsmokers are shown in (a), hyperglycemic nonsmokers in (b), normoglycemic smokers in (c) and hyperglycemic smokers in (d). Each network graph contains nodes (circles sized by relative abundance per group) and edges (lines). Nodes represent species-level OTU's and edges represent Spearman's $\rho$. Edges are colored green for positive correlation and red for negative correlation. Only significant correlations $(P<0.05, t$-test $)$ with a coefficient of at least 0.75 are shown. Network anchors are highlighted in red font.

Dialister, Eubacterium, Fusobacterium, Atopobium, Alloprevotella and Porphyromonas and higher levels of facultatives (for example, Hemophilus, Pseudomonas, Neisseria) when compared to smokers $(P<0.05$, Figure $4 \mathrm{~b})$. While smokers demonstrated lower levels of facultatives and higher abundances of anaerobes when compared to normoglycemics, diabetics exhibited the exact opposite distributions (Figure 2d). The core microbiome of periodontitis in smokers was larger than that of diabetics $(22 \%$ of s-OTUs in smokers and $17 \%$ in diabetics, Figure 3a). Moreover, the core species in smokers accounted for $85 \%$ of the overall abundance. By contrast, only $32 \%$ of the overall abundance in diabetics was contributed by the core species. Co-occurrence was sparse and congeneric in smokers, and robust inter-generic microbial hubs were seen in diabetics (Figure 5).

To compare the ecological shift from health to disease between smokers and diabetics, the microbiomes of smokers with and without periodontitis were analyzed and compared to periodontally healthy nonsmokers (controls). In contrast to diabetics, PCoA and LDA revealed distinct clustering of 

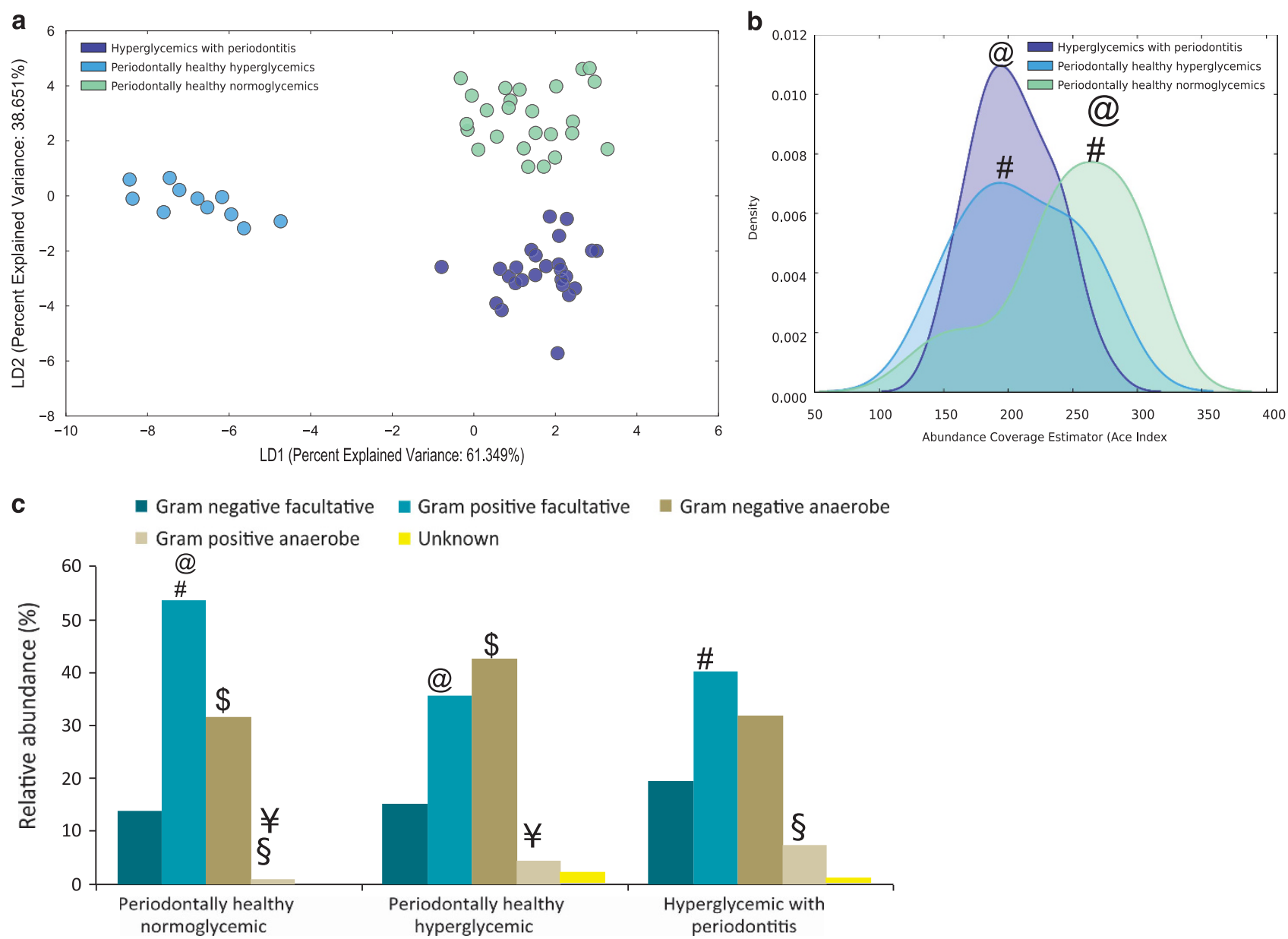

Figure 6 Ecological shifts from health to disease in hyperglycemics. (a) shows the LDA plot of periodontally healthy normoglycemics, periodontally healthy hyperglycemics and hyperglycemic individuals with periodontitis $(P=0.01$, Manova Wilks test). Kernel density curves of alpha diversity (ACE) are shown in (b) (The peak indicates the median values for each group, and the $x$ axis shows the data range) and distribution of s-OTUs by gram staining characteristics and oxygen requirements in (c). Groups that share the same symbol are significantly different from each other $(P<0.05$, Kruskal-Wallis test).

groups $(P=0.01)$ based on smoking status but not disease status (Figure 7a). Smokers with periodontitis exhibited significantly lower species richness than both periodontally healthy smokers and nonsmokers $(P=0.00003$, Figure 7b). Both periodontally healthy smokers and smokers with periodontitis exhibited significantly lower levels of facultatives and higher levels of anaerobes when compared to periodontally healthy nonsmokers $(P=0.005$, Figure 7c). Similar to diabetics, periodontally healthy smokers shared only a small fraction $(29 \%)$ of their core microbiome with healthy nonsmokers (Figure 3c). However, unlike diabetics, smokers with periodontitis shared $86 \%$ of their microbiome with periodontally healthy smokers.

In summary, while certain community trends (low inventory diversity, periodontitis-associated signatures in periodontal health) were similar between smoking and diabetes, these communities demonstrated significant differences based on the environmental perturbation. For instance, the microbial profile was significantly more homogenous among smokers than diabetics, as evidenced by the larger core microbiome and the inclusion of numerically dominant species in the core in the former. Thus, while differences among individuals who smoke were due to species that together accounted for $<15 \%$ of the overall abundance (the 'rare biosphere'), in diabetics, numerically dominant species varied significantly among individuals, contributing to significant inter-individual variation or heterogeneity. Smokers demonstrated lower levels of anaerobes and higher levels of aerobes when compared to normoglycemics, on the other hand, diabetics demonstrated the exact opposite trend. Co-occurrence appeared to be driven by interbacterial interactions in smokers, and by the microenvironment in diabetics. Moreover, transition from health to disease was not accompanied by microbial shifts in smokers; in diabetics, transition from health to disease was associated with introduction of new members and loss of others. 
a

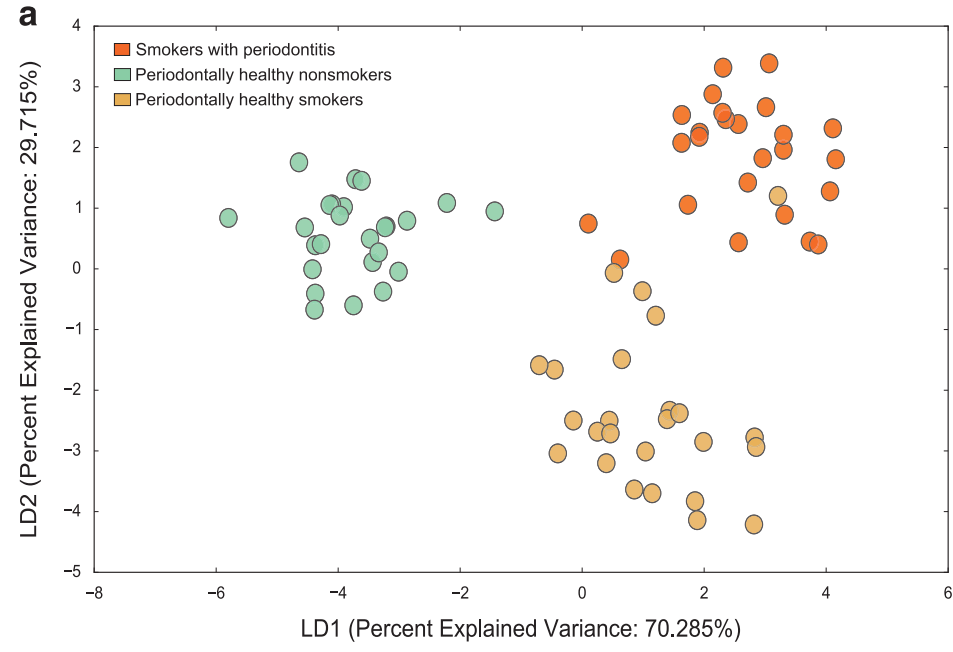

b

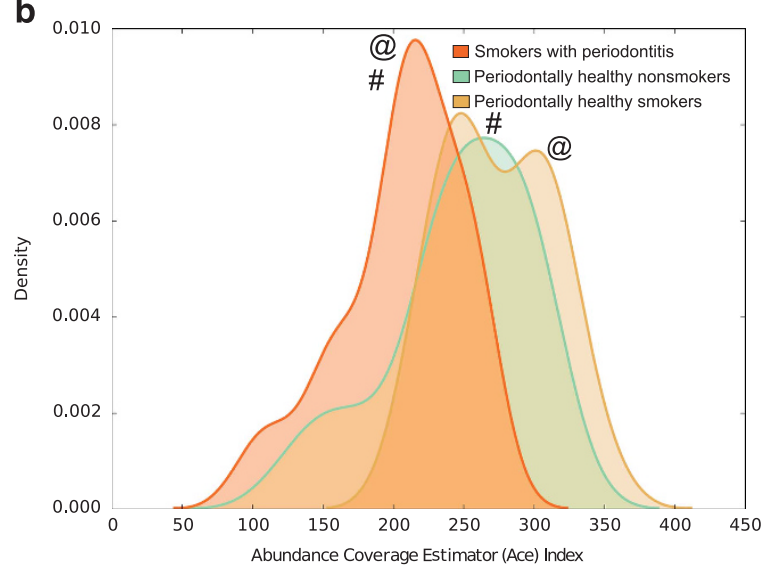

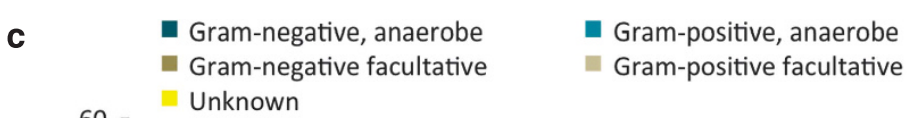

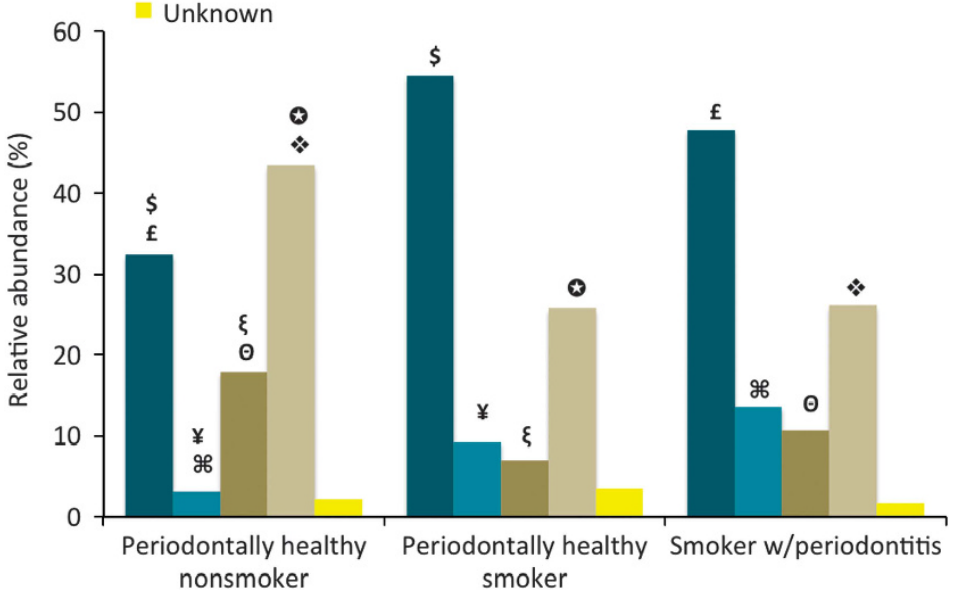

Figure 7 Ecological shifts from health to disease in smokers. (a) shows the LDA plot of periodontally healthy nonsmokers, periodontally healthy smokers and smokers with periodontitis $(P=0.008$, Manova Wilks test). Kernel density curves of alpha diversity (ACE) are shown in (b) (The peak indicates the median values for each group, and the $x$ axis shows the data range) and distribution of s-OTUs by gram staining characteristics and oxygen requirements in (c). Groups that share the same symbol are significantly different from each other $(P<0.05$, Kruskal-Wallis test).

A tale of two risks-the combined effect of smoking and diabetes is greater than the sum of the parts

To investigate the combined effects of diabetes and smoking on the subgingival microbiome, we compared the microbial assemblages of uncontrolled hyperglycemics with a 10 pack-year smoking history to normoglycemic smokers and hyperglycemic nonsmokers. All these individuals had periodontitis; unlike the smokers, diabetics and controls, we were unable to identify periodontally healthy diabetic smokers. PCoA as well as LDA revealed a significant clustering of the three groups $(P=0.001$, Figure 2a). Diabetic-smokers also demonstrated significantly lower alpha diversity (median of $150, P=0.0001$ ), a bimodal distribution of the density plot and a significantly greater range in the ACE index when compared to diabetics or smokers (Figure 2b). Additionally, the relative risk for diabetic smokers to demonstrate an ACE index $<150$ was 11 , while it was 2.1 for smokers and 4.7 for diabetics. Diabetic- smokers exhibited lower levels of gram-negative anaerobes and higher levels of gram-negative facultatives when compared to both smokers and diabetics $(P<0.05$, Figure 2c). The microbiomes of 23 diabetic smokers contained gram-negative facultatives at $10 \%$ or more of the total microbiota (and gram-negative anaerobes at $15 \%$ or less), while six diabetics and two smokers had the same numbers. These species belonged to Leptotrichia, Pseudomonas, Acinetobacter, Brevundimonas, Enterobacter, Alloprevotella, Bergeyella, Terrahemophilus and Stenotophomonas $(P<0.05$, Figures $4 \mathrm{c}$ and d). The core microbiome in diabetic smokers was the smallest $4 \%$ of s-OTUs in diabetic smokers when compared to $30 \%$ in controls, $23 \%$ in smokers, and $18 \%$ in diabetics, Figure 3a). However, microbial species in diabetic smokers demonstrated the most robust co-occurrence patterns, with over $150 \mathrm{~s}$-OTUs contributing to the creation of microbial hubs (Figure 5d). Taken together, the relative risk of 
having a lower diversity, higher levels of gramnegative facultatives, lower levels of gram-negative anaerobes and a smaller core are much higher in diabetic smokers than would be expected by adding the odds of smokers and diabetics, suggesting that when these presses intersect, their effect is multiplicative, not additive.

\section{Discussion}

The association between type 2 diabetes and the subgingival microbiome has previously been studied using cultivation-based or targeted molecular approaches (Zambon et al., 1988; Hintao et al., 2007; Ebersole et al., 2008). These investigations concluded that hyperglycemia does not influence the subgingival microbiome (Taylor et al., 2013). However, when we examined this ecosystem by combining a rigorous case-control clinical study design with a comprehensive microbiomics approach for community characterization, we found evidence to support the hypothesis that specific environmental presses influence both community structure and membership in the subgingival ecosystem in distinctive ways.

In the present investigation, the biggest differences in both smokers and diabetics were between healthy and diseased subjects, not between healthy and diseased sites in the same individual. Although early investigations suggested that shallow sites might be more closely aligned to health rather than with disease (Kumar et al., 2005), recent investigations using deep sequencing methodology suggest that periodontitis may result from a whole-mouth dysbiosis rather than site-specific changes (Abusleme et al., 2013). The data presented here thus corroborate newly emerging evidence.

Periodontitis is a chronic disease, and the immuno-inflammatory effects of a dysbiotic microbiome take several years to manifest as clinically measurable disease. Therefore, it is challenging to establish cause and effect using a traditional prospective study design. In an effort to overcome this, we have used a case-control study design with both environmental drivers (diabetes and smoking) as well as periodontal health status (periodontal health and periodontitis) as variables. However, the oral cavity is an open microbial ecosystem with allochthonous and autochthonous members, and one of the greatest limitations of a cross sectional study design is the inability to distinguish between these two constituents. We have used two complementary strategies to surmount this issue. We defined the core microbiome of each group as the suite of species identified in $\geqslant 80 \%$ of subjects and compared groups based on species that have been selected for by environmental drivers; a strategy that allows for comparisons of stable associations between groups. This approach was first delineated by the Human Microbiome Project (Human
Microbiome Project C, 2012) and is now widely used in ecological investigations (Backhed et al., 2012; Shade and Handelsman, 2012; D Ainsworth et al., 2015). Diabetic smokers, however, demonstrated extreme microbial heterogeneity, with only $4 \%$ of the microbial suite common to the majority of the cohort. Hence, as a second strategy, we used network analysis of co-occurrence patterns to identify species that are niche-partners in each environment; drawing on similar methods used in other ecosystems (Barberan et al., 2012; Faust et al., 2012).

An interesting finding was that, in contrast to the sparse congeneric clusters seen in normoglycemics and smokers with periodontitis, both diabetic nonsmokers and smokers demonstrated dense hubs made up of both congeneric and inter-generic networks. Congeneric species have a smaller evolutionary divergence and therefore, share similar morphological and ecological characteristics (Sfenthourakis et al., 2006). Hence, congeneric clusters are the norm in most niches. Inter-generic clusters, on the other hand, may be driven by two factors: (i) the inherent characteristics of the microenvironment to support organisms with similar lifestyle requirements (namely, oxygen tension, $\mathrm{pH}$, redox potential and nutrient supply) and (ii) metabolic, structural and nutritional interbacterial partnerships. Taken together with the finding that diabetics demonstrated significant microbial heterogeneity, the data suggest that the hyperglycemic microenvironment enforces habitat filtering, favoring organisms that thrive under glucoserich, pro-oxidant, protein-rich and anaerobic conditions.

This hypothesis finds further support in the significantly elevated levels of Capnocytophaga, Pseudomonas, Bergeyella, Sphingomonas, Corynebacterium, Propionibacterium, and Neisseria in hyperglycemic individuals. Capnocytophaga have been previously identified in high numbers in periodontitis in diabetics (Ciantar et al., 2005), and these individuals demonstrate depressed IgG antibody profiles to these species (Dyer et al., 1997). Capnocytophaga also exhibit profuse growth in the presence of glucose (Spratt et al., 1996). Pseudomonads have been implicated in the etiology of diabetic foot infections (Muthu et al., 2006) and otitis media (Zaky et al., 1976); and glycemic levels are a critical determinant of bacterial burden in such infections (Gill et al., 2016). Similar lines of evidence implicate Corynebacterium, Propionibacterium and Sphingomonas as causative organisms in polymicrobial skin and mucosal infections in diabetics (Pozzilli and Leslie, 1994). Neisseria are well known for ironpiracy (Noinaj et al., 2012); they extract it directly from human transferrin, an iron transport protein that is pathologically elevated in diabetics (Memisogullari and Bakan, 2004). Pseudomonas, Neisseria and Propionibacterium are well-known stimulators of matrix metallo-proteinases (MMPs) 
(Leppert et al., 2000; Miyajima et al., 2001; Lee et al., 2010), an enzyme that plays a critical role in periodontal destruction. An important finding was that this shift in community membership and structure was evident even in the absence of clinical inflammation and periodontal destruction in diabetics, suggesting that these species may play a role in increasing susceptibility to periodontitis in these individuals.

Periodontitis is a significant public health concern, not only because the costs of treating this disease and its sequelae are comparable to outpatient treatment of cardiac and metabolic diseases (Gjermo and Grytten, 2009; Fardal et al., 2012; Mohd-Dom et al., 2014), but also because of its role in the pathogenesis of several life-threatening diseases, including cerebrovascular accidents and atherosclerosis (Darveau et al., 1997; D'Aiuto et al., 2005). Hence, risk assessment is a critical element in disease prevention and successful therapeutic intervention. Our findings that smoking and diabetes affect the microbiome in distinct ways and that the synergistic impact of these two factors varies significantly from either in isolation underscores the need for developing a personalized risk assessment strategy that assigns differential weightage to each risk factor. It also underscores the importance of integrating microbial community-level metrics into periodontal risk assessments, rather than targeting individual species.

\section{Conflict of Interest}

The authors declare no conflict of interest.

\section{Acknowledgements}

The sequences have been deposited in the Sequence Read Archive of the NCBI (Accession number: SRP090878). These studies were funded through National Institute of Dental and Craniofacial Medicine (NIDCR) grant R01DE022579 and through American Society for Microbiology grant (ASM-IUSSTF Professorship) to Vinayak Joshi. Shareef Dabdoub was supported by NIDCR grant T32DE014320.

\section{Ethical statement}

The study was approved by the Office of Research at The Ohio State University (2008H0122) and the Ethics Committee of Rajiv Gandhi University, India and was conducted in accordance with the approved guidelines. All subjects were recruited to the study after informed consent was obtained.

\section{Author contributions}

SMG was part of the clinical team, participated in the sequence analysis and drafted the manuscript. VJ and NRD carried out the clinical examinations and sample collection. MLF contributed to the sequence and the data analysis. SMD carried out the bioinformatics analysis. HNN participated in the design of the study and oversaw the statistical analysis. BO'D contributed to the clinical study design and served as a consultant for the clinical analysis. PSK conceived of the study, and participated in its design and coordination and helped to draft the manuscript. All authors read and approved the final manuscript.

\section{References}

Abusleme L, Dupuy AK, Dutzan N, Silva N, Burleson JA, Strausbaugh LD et al. (2013). The subgingival microbiome in health and periodontitis and its relationship with community biomass and inflammation. ISME J 7: 1016-1025.

American Diabetes Association. (2015). (2) Classification and diagnosis of diabetes. Diabetes Care 38: S8-S16.

Armitage GC. (1999). Development of a classification system for periodontal diseases and conditions. Ann Periodontol 4: 1-6.

Backhed F, Fraser CM, Ringel Y, Sanders ME, Sartor RB, Sherman PM et al. (2012). Defining a healthy human gut microbiome: current concepts, future directions, and clinical applications. Cell Host Microbe 12: 611-622.

Barberan A, Bates ST, Casamayor EO, Fierer N. (2012). Using network analysis to explore co-occurrence patterns in soil microbial communities. ISME $J$ 6: 343-351.

Bastian M, Heymann S, Jacomy M. (2009). Gephi: an open source software for exploring and manipulating networks. ICWSM 8: 361-362.

Bergstrom J, Eliasson S, Dock J. (2000). A 10-year prospective study of tobacco smoking and periodontal health. J Periodontol 71: 1338-1347.

Caporaso JG, Kuczynski J, Stombaugh J, Bittinger K, Bushman FD, Costello EK et al. (2010). QIIME allows analysis of high-throughput community sequencing data. Nat Methods 7: 335-336.

Casarin RC, Barbagallo A, Meulman T, Santos VR, Sallum EA, Nociti FH et al. (2013). Subgingival biodiversity in subjects with uncontrolled type-2 diabetes and chronic periodontitis. J Periodontal Res 48: $30-36$.

CDC. (2014). National diabetes statistics report: estimates of diabetes and its burden in the United States, 2014. CDC: Atlanta, GA, USA.

Chen FM, Gao LN, Tian BM, Zhang XY, Zhang YJ, Dong GY et al. (2005) Position paper: epidemiology of periodontal diseases. J periodontol 76: 1406-1419.

Chen T, Yu WH, Izard J, Baranova OV, Lakshmanan A, Dewhirst FE. (2010). The Human Oral Microbiome Database: a web accessible resource for investigating oral microbe taxonomic and genomic information. Database 2010: baq013.

Ciantar M, Gilthorpe MS, Hurel SJ, Newman HN, Wilson M, Spratt DA. (2005). Capnocytophaga spp. in periodontitis patients manifesting diabetes mellitus. J Periodontol 76: 194-203.

Clarke NG, Shephard BC, Hirsch RS. (1981). The effects of intra-arterial epinephrine and nicotine on gingival circulation. Oral Surg Oral Med Oral Pathol 52: 577-582.

D Ainsworth T, Krause L, Bridge T, Torda G, Raina J-B, Zakrzewski $\mathrm{M}$ et al. (2015). The coral core microbiome identifies rare bacterial taxa as ubiquitous endosymbionts. ISME J 9: 2261-2274. 
D'Aiuto F, Graziani F, Tete S, Gabriele M, Tonetti MS. (2005). Periodontitis: from local infection to systemic diseases. Int J Immunopathol Pharmacol 18: 1-11.

Dabdoub SM, Fellows ML, Paropkari AD, Mason MR, Huja SS, Tsigarida AA et al. (2016). PhyloToAST: Bioinformatics tools for species-level analysis and visualization of complex microbial datasets. Sci Rep 6: 29123.

Darveau RP, Tanner A, Page RC. (1997). The microbial challenge in periodontitis. Periodontol 2000 14: 12-32.

Delima SL, McBride RK, Preshaw PM, Heasman PA, Kumar PS. (2010). Response of subgingival bacteria to smoking cessation. J Clin Microbiol 48: 2344-2349.

Dyer JK, Peck MA, Reinhardt RA, Duckworth WC, Booth SJ, Seymour GJ et al. (1997). HLA-D types and serum IgG responses to Capnocytophaga in diabetes and periodontitis. J Dent Res 76: 1825-1832.

Ebersole JL, Holt SC, Hansard R, Novak MJ. (2008). Microbiologic and immunologic characteristics of periodontal disease in Hispanic americans with type 2 diabetes. J Periodontol 79: 637-646.

Eke PI, Dye BA, Wei L, Thornton-Evans GO, Genco RJ, Cdc Periodontal Disease Surveillance workgroup: James Beck GDRP. (2012). Prevalence of periodontitis in adults in the United States: 2009 and 2010. J Dent Res 91: 914-920.

Fardal O, O'Neill C, Gjermo P, Fardal E, Sandvik L, Hansen BF et al. (2012). The lifetime direct cost of periodontal treatment: a case study from a Norwegian specialist practice. J Periodontol 83: 1455-1462.

Faust K, Sathirapongsasuti JF, Izard J, Segata N, Gevers D, Raes J et al. (2012). Microbial co-occurrence relationships in the human microbiome. PLoS Comput Biol 8: e1002606.

Ford ES, Mokdad AH, Gregg EW. (2004). Trends in cigarette smoking among US adults with diabetes: findings from the Behavioral Risk Factor Surveillance System. Prev Med 39: 1238-1242.

Fullmer SC, Preshaw PM, Heasman PA, Kumar PS. (2009). Smoking cessation alters subgingival microbial recolonization. J Dent Res 88: 524-528.

Gill SK, Hui K, Farne H, Garnett JP, Baines DL, Moore LS et al. (2016). Increased airway glucose increases airway bacterial load in hyperglycaemia. Sci Rep 6: 27636.

Gjermo PE, Grytten J. (2009). Cost-effectiveness of various treatment modalities for adult chronic periodontitis. Periodontol 2000 51: 269-275.

Gomes SC, Piccinin FB, Oppermann RV, Susin C, Marcantonio RA. (2009). The effect of smoking on gingival crevicular fluid volume during the treatment of gingivitis. Acta Odontol Latinoam 22: 201-206.

Goodson JM, Tanner AC, Haffajee AD, Sornberger GC, Socransky SS. (1982). Patterns of progression and regression of advanced destructive periodontal disease. J Clin Periodontol 9: 472-481.

Griffen AL, Beall CJ, Campbell JH, Firestone ND, Kumar PS, Yang ZK et al. (2012). Distinct and complex bacterial profiles in human periodontitis and health revealed by $16 \mathrm{~S}$ pyrosequencing. ISME J 6: 1176-1185.

Hagberg AA, Schult DA, Swart PJ. (2008). Exploring network structure, dynamics, and function using NetworkX. Proceedings of the 7th Python in Science Conferences (SciPy 2008), 19-24 August 2008. Gäel Varoquaux, Travis Vaught, and Jarrod Millman: Pasadena, CA, USA, 2008, pp 11-16.

Hammond EC, Selikoff IJ, Seidman H. (1979). Asbestos exposure, cigarette smoking and death rates. Ann NY Acad Sci 330: 473-490.
Hanioka T, Tanaka M, Takaya K, Matsumori Y, Shizukuishi S. (2000). Pocket oxygen tension in smokers and non-smokers with periodontal disease. $J$ Periodontol 71: 550-554.

Hintao J, Teanpaisan R, Chongsuvivatwong V, Ratarasan C, Dahlen G. (2007). The microbiological profiles of saliva, supragingival and subgingival plaque and dental caries in adults with and without type 2 diabetes mellitus. Oral Microbiol Immunol 22: 175-181.

Human Microbiome Project C. (2012). Structure, function and diversity of the healthy human microbiome. Nature 486: 207-214.

Kassebaum NJ, Bernabe E, Dahiya M, Bhandari B, Murray CJ, Marcenes W. (2014). Global burden of severe periodontitis in 1990-2010: a systematic review and meta-regression. J Dent Res 93: 1045-1053.

Kumar PS, Matthews CR, Joshi V, de Jager M, Aspiras M. (2011a). Tobacco smoking affects bacterial acquisition and colonization in oral biofilms. Infect Immun 79: 4730-4738.

Kumar PS. (2012). Smoking and the subgingival ecosystem: a pathogen-enriched community. Future Microbiol 7: 917-919.

Kumar PS, Griffen AL, Moeschberger ML, Leys EJ. (2005). Identification of candidate periodontal pathogens and beneficial species by quantitative $16 \mathrm{~S}$ clonal analysis. J Clin Microbiol 43: 3944-3955.

Kumar PS, Brooker MR, Dowd SE, Camerlengo T. (2011b). Target region selection is a critical determinant of community fingerprints generated by $16 \mathrm{~S}$ pyrosequencing. PLoS One 6: e20956.

Larsen N, Vogensen FK, van den Berg FWJ, Nielsen DS, Andreasen AS, Pedersen BK et al. (2010). Gut Microbiota in Human Adults with Type 2 Diabetes Differs from Non-Diabetic Adults. PLoS One 5: e9085.

Lee SE, Kim J-M, Jeong SK, Jeon JE, Yoon H-J, Jeong M-K et al. (2010). Protease-activated receptor-2 mediates the expression of inflammatory cytokines, antimicrobial peptides, and matrix metalloproteinases in keratinocytes in response to Propionibacterium acnes. Arch Dermatol Res 302: 745-756.

Leppert D, Leib S, Grygar C, Miller K, Schaad U, Holländer G. (2000). Matrix metalloproteinase (MMP)-8 and MMP-9 in cerebrospinal fluid during bacterial meningitis: association with blood-brain barrier damage and neurological sequelae. Clin Infect Dis 31: 80-84.

Letunic I, Bork P. (2011). Interactive Tree Of Life v2: online annotation and display of phylogenetic trees made easy. Nucleic Acids Res 39: W475-W478.

Loe H, Silness J. (1963). Periodontal disease in pregnancy. i. prevalence and severity. Acta Odontol Scand 21: 533-551.

Loe H. (1993). Periodontal disease. The sixth complication of diabetes mellitus. Diabetes Care 16: 329-334.

Love MI, Huber W, Anders S. (2014). Moderated estimation of fold change and dispersion for RNA-seq data with DESeq2. Genome Biol 15: 550.

Mason MR, Preshaw PM, Nagaraja HN, Dabdoub SM, Rahman A, Kumar PS. (2015). The subgingival microbiome of clinically healthy current and never smokers. ISME J 9: 268-272.

Mavropoulos A, Aars H, Brodin P. (2003). Hyperaemic response to cigarette smoking in healthy gingiva. J Clin Periodontol 30: 214-221. 
McArdle BH, Anderson MJ. (2001). Fitting multivariate models to community data: A comment on distancebased redundancy analysis. Ecology 82: 290-297.

Memisogullari R, Bakan E. (2004). Levels of ceruloplasmin, transferrin, and lipid peroxidation in the serum of patients with Type 2 diabetes mellitus. J Diabetes Complications 18: 193-197.

Miyajima S, Akaike T, Matsumoto K, Okamoto T, Yoshitake J, Hayashida K et al. (2001). Matrix metalloproteinases induction by pseudomonal virulence factors and inflammatory cytokines in vitro. Microb Pathog 31: 271-281.

Mohd-Dom T, Ayob R, Mohd-Nur A, Abdul-Manaf MR, Ishak N, Abdul-Muttalib K et al. (2014). Cost analysis of periodontitis management in public sector specialist dental clinics. BMC Oral Health 14: 56.

Muthu SE, Aberna RA, Mohan V, Premalatha G, Srinivasan RS, Thyagarajan SP et al. (2006). Phenotypes of isolates of Pseudomonas aeruginosa in a diabetes care center. Arch Med Res 37: 95-101.

$\mathrm{Ng}$ M, Freeman MK, Fleming TD, Robinson M, Dwyer-Lindgren L, Thomson B et al. (2014). Smoking prevalence and cigarette consumption in 187 countries, 1980-2012. Jama 311: 183-192.

Noinaj N, Easley NC, Oke M, Mizuno N, Gumbart J, Boura E et al. (2012). Structural basis for iron piracy by pathogenic Neisseria. Nature 483: 53-58.

Ohlrich EJ, Cullinan MP, Leichter JW. (2010). Diabetes, periodontitis, and the subgingival microbiota. J Oral Microbiol 2.

Paropkari AD, Leblebicioglu B, Christian LM, Kumar PS. (2016). Smoking, pregnancy and the subgingival microbiome. Sci Rep 6: 30388.

Pozzilli P, Leslie RDG. (1994). Infections and diabetes: mechanisms and prospects for prevention. Diabetic Med 11: 935-941.

Sfenthourakis S, Tzanatos E, Giokas S. (2006). Species co-occurrence: the case of congeneric species and a causal approach to patterns of species association. Glob Ecol Biogeogr 15: 39-49.

Shade A, Handelsman J. (2012). Beyond the Venn diagram: the hunt for a core microbiome. Environ Microbiol 14: 4-12.

Shchipkova AY, Nagaraja HN, Kumar PS. (2010a). Subgingival microbial profiles in smokers with periodontitis. J Dental Res 89: 1247-1253.

Shchipkova AY, Nagaraja HN, Kumar PS. (2010b). Subgingival microbial profiles of smokers with periodontitis. J Dental Res 89: 1247-1253.
Spratt DA, Greenman J, Schaffer AG. (1996). Capnocytophaga gingivalis: effects of glucose concentration on growth and hydrolytic enzyme production. Microbiology 142(Pt 8): 2161-2164.

Taylor JJ, Preshaw PM, Lalla E. (2013). A review of the evidence for pathogenic mechanisms that may link periodontitis and diabetes. J Clin Periodontol 40(Suppl 14): S113-S134.

Tomar SL, Asma S. (2000). Smoking-attributable periodontitis in the United States: findings from NHANES III. National Health and Nutrition Examination Survey. J Periodontol 71: 743-751.

Wu J, Peters BA, Dominianni C, Zhang Y, Pei Z, Yang L et al. (2016). Cigarette smoking and the oral microbiome in a large study of American adults. ISME J 10: 2436-2446.

Yamaguchi M, Takada R, Kambe S, Hatakeyama T, Naitoh K, Yamazaki K et al. (2005). Evaluation of time-course changes of gingival crevicular fluid glucose levels in diabetics. Biomed Microdevices 7: 53-58.

Zaky DA, Bentley DW, Lowy K, Betts RF, Douglas RG. (1976). Malignant external otitis: A severe form of otitis in diabetic patients. Am J Med 61: 298-302.

Zambon JJ, Reynolds H, Fisher JG, Shlossman M, Dunford R, Genco RJ. (1988). Microbiological and immunological studies of adult periodontitis in patients with noninsulin-dependent diabetes mellitus*. J Periodontol 59: 23-31.

Zhou M, Rong R, Munro D, Zhu C, Gao X, Zhang Q et al. (2013). Investigation of the effect of type 2 diabetes mellitus on subgingival plaque microbiota by highthroughput $16 \mathrm{~S}$ rDNA pyrosequencing. PLoS One 8: e61516.

(c) (1) () $\odot$ This work is licensed under a Creative Commons Attribution-NonCommercialNoDerivs 4.0 International License. The images or other third party material in this article are included in the article's Creative Commons license, unless indicated otherwise in the credit line; if the material is not included under the Creative Commons license, users will need to obtain permission from the license holder to reproduce the material. To view a copy of this license, visit http://creativecommons.org/ licenses/by-nc-nd/4.0/

(C) The Author(s) 2017 